\title{
Produção e caracterização físico-química de geleia de menta enriquecida com
}

\section{spirulina (Spirulina platensis)}

\author{
Production and physical and chemical characterization of mented jelly enriched with spirulina
}

(Spirulina platensis)

Producción y caracterización física y química de jalea mentada enriquecida con espirulina

(Spirulina platensis)

\section{Resumo}

Existe uma grande preocupação com um estilo de vida mais saudável por uma parte crescente dos indivíduos. Nesse contexto, suplementos vitamínicos e alimentos enriquecidos figuram como possibilidades práticas de potencializadores nutritivos. A Spirulina Platensis apresenta alta concentração de nutrientes e é um dos mais completos suplementos naturais disponíveis na natureza. Sendo assim, a produção de um alimento com boa aceitabilidade, como a geleia, figura como uma alternativa para suplementar a Spirulina, haja vista que o cheiro e cor forte de sua forma não processada muitas vezes atrapalham a sua utilização na alimentação. Com esse objetivo, foi elaborada no presente estudo uma geleia de menta enriquecida com Spirulina (Spirulina platensis) em diferentes formulações. Para averiguação das formulações foram feitos teste físico-químicos (teor de água e sua atividade, cinzas, Sólidos solúveis, proteína e acidez) e microbiológicos, estes últimos seguiram o preconizado pela legislação vigente. As características físico-químicas se mostraram enquadradas nos padrões estabelecidos pela legislação, fator que contribui para a formação de aspectos sensoriais agradáveis e desejáveis, tornando a geleia viável para consumo. Foram obtidos também resultados positivos nos estudos microbiológicos, onde os resultados estavam dentro dos parâmetros adequados, evidenciando a qualidade das geleias, da matéria-prima e do processo produtivo. Tendo em vista tais dados, pode-se concluir que a geleia deste 
estudo tem um alto teor nutricional, podendo tornar-se futuramente uma grande auxiliar na suplementação nutricional da população.

Palavras-chave: Geleia; Tecnologia dos alimentos; Análise microbiológica.

\begin{abstract}
There is a great concern with a healthier lifestyle for an increasing number of individuals, which is reflected in an increase in life expectancy. In this context, vitamin supplements and enriched foods appear as practical possibilities for nutritional enhancers. Spirulina Platensis has a high concentration of nutrients and is one of the most complete natural supplements available in nature. Thus, the production of a food with good acceptability, such as jelly, appears as an alternative to supplement Spirulina, given that the smell and strong color of its unprocessed form often hinder its use in food. For this purpose, a mint jelly enriched with Spirulina (Spirulina platensis) in different formulations was prepared in the present study. In order to verify the formulations, physical-chemical tests (water content and its activity, ashes, soluble solids, protein and acidity) and microbiological tests were carried out, the latter followed the recommendations of current legislation. The physical-chemical characteristics were shown to conform to the standards established by the legislation, a factor that contributes to the formation of pleasant and desirable sensory aspects, making the jelly viable for consumption. Positive results were also obtained in microbiological studies, where the results were within the appropriate parameters, showing the quality of the jellies, the raw material and the production process. In view of such data, it can be concluded that the jelly in this study has a high nutritional content, and may in the future become a great help in the nutritional supplementation of the population.
\end{abstract}

Keywords: Jelly; Food technology; Microbiological analysis.

\title{
Resumen
}

Existe una gran preocupación por un estilo de vida más saludable para un número creciente de personas, lo que se refleja en un aumento de la esperanza de vida. En este contexto, los complementos vitamínicos y los alimentos enriquecidos aparecen como posibilidades prácticas de potenciadores nutricionales. Spirulina Platensis tiene una alta concentración de nutrientes y es uno de los suplementos naturales más completos disponibles en la naturaleza. Así, la producción de un alimento con buena aceptabilidad, como la jalea, aparece como una alternativa al complemento de la espirulina, dado que el olor y el color fuerte de su forma no procesada dificultan a menudo su uso en alimentos. Para este propósito, en el presente estudio se preparó una gelatina de menta enriquecida con Spirulina (Spirulina platensis) en diferentes formulaciones. Para la verificación de las formulaciones se realizaron pruebas físico-químicas (contenido de agua y su actividad, cenizas, sólidos solubles, proteína y acidez) y pruebas microbiológicas, estas últimas siguieron las recomendaciones de la legislación vigente. Se demostró que las características físico-químicas se ajustan a los estándares establecidos por la legislación, factor que contribuye a la formación de aspectos sensoriales agradables y deseables, haciendo viable la jalea para el consumo. También se obtuvieron resultados positivos en estudios microbiológicos, donde los resultados estuvieron dentro de los parámetros adecuados, mostrando la calidad de las jaleas, la materia prima y el proceso de producción. A la vista de tales datos, se puede concluir que la gelatina de este estudio tiene un alto contenido nutricional, pudiendo en el futuro convertirse en una gran ayuda en la suplementación nutricional de la población.

Palabras clave: Gelatina; Tecnología de los alimentos; Análisis microbiológico.

\section{Introdução}

Após a revolução agrícola, houve uma expressiva modificação na relação entre o homem e o alimento, a qual acentuouse ainda mais na Época Moderna. Esse fato traduz-se na oferta de uma grande diversidade de produtos para o consumo humano, com suas diferentes composições e propósitos (Silva \& Silva, 2013).

Existe uma preocupação com um estilo de vida mais saudável por uma parte crescente dos indivíduos, a qual se reflete em um aumento da expectativa de vida. Nesse contexto, suplementos vitamínicos e alimentos enriquecidos figuram como possibilidades práticas de potencializadores nutritivos para a população geral (Abe-matsumoto et al., 2015).

Muitos alimentos estão sendo pesquisados para otimizar a suplementação, podendo-se observar como exemplo a geleia (Krolow, 2013). Segundo a Legislação Brasileira, este deve ser um produto obtido da concentração da polpa ou suco de frutas com quantidades adequadas de açúcar e pectina, que depois de cozido e frio deve ter consistência branda, compacta e trêmula. As geleias artesanais, além de fornecerem sais minerais inerentes às frutas que forem colocadas na sua preparação, geralmente são ricas também em vitaminas. Quando são adicionados pedaços de frutas à geleia, este produto passa a chamar-se de "geleiada" (Brasil, 1978). 
A geleia pode ser incrementada com o uso de outros constituintes em sua formulação, como a Mentha haplocalyx. Trata-se de uma erva perene da família Labiaceae, que apresenta propriedades alimentares, assim como também pode ser utilizada em tratamentos médicos e fabricação de cosméticos. Foi demonstrado pela medicina tradicional que há benefícios quando ela é administrada para tratar constipações, tosse, febre, e também indigestão. Além disso, a farmacologia têm encontrado várias propriedades na $M$. haplocalyx, tais como função antioxidante, anti-inflamatória, atividade antiviral, antitumoral e antidiabetes (Fang et al., 2020).

Outro produto que pode ser utilizado na elaboração deste alimento é a Spirulina platensis. Trata-se de uma alga microscópica azul-verde utilizada como complemento alimentar devido ao seu elevado valor nutricional (60\%-70\% de proteína), bem como à sua grande quantidade de vitaminas, minerais, aminoácidos essenciais, ácidos poliinsaturados, ômega-3 e ômega-6 (Richmond, 2004). Além disso, em condições experimentais, exibe muitas propriedades farmacológicas, incluindo características antioxidantes, anti-inflamatórias e imunomoduladoras (Abdel-daim et al., 2013).

Estas algas apresentam teor proteico superior a qualquer outra fonte alimentar, apresentando concentração de aminoácidos essenciais acima do padrão sugerido pela FAO/1985 - Food and Agriculture Organization of the United Nations (Bertoldi et al., 2008). A sua importância é tal que a S. platensis já foi utilizada como fonte de alimento e suplemento primordial em programas espaciais da National Aeronautics and Space Administration (NASA), agência do Governo americano encarregada de pesquisa e desenvolvimento da exploração espacial (Oliveira et al., 2013).

As paredes celulares da Spirulina são similares às das bactérias gram-positivas, consistindo no polissacarídeo glicosamina e em ácido murâmico associados a peptídeos, não contendo celulose como as macroalgas. Os polissacarídeos extracelulares ou exopolissacarídeos (EPS), sintetizados por cianobactérias, podem ser facilmente recuperados e possuem alto potencial de utilização pela indústria farmacêutica, cosmética e alimentícia como estabilizantes, emulsificantes e espessantes (Philippis et al., 2001).

Lisboa et al. (2016) confirmam, em seu trabalho, que a biomassa de Spirulina sp. LEB 18 possui uma alta concentração de proteína e as condições necessárias para cultivá-la podem ser alteradas para estimular uma maior concentração desse bioproduto. A concentração de proteína $(51,66 \%)$ foi usada para calcular a quantidade de biomassa necessária para obter as suspensões de cada ensaio.

Segundo Falcão et al. (2020), que desenvolveram uma Geleia de umbu (S. tuberosa) enriquecida com proteínas pela adição de Spirulina sp. LEB-18, a utilização da microalga pode potencializar o valor nutricional e agregar benefícios à saúde quando incorporada a diferentes produtos da indústria de alimentos, principalmente como ingrediente funcional enriquecido em proteínas.

Para o mercado de alimentos, a criação de novos produtos torna-se essencial, aproveitando o ensejo da exigência dos consumidores que almejam estas inovações. Sendo assim, o campo da alimentação que apresenta maior elevação é aquele que agrega algum benefício de saúde ao produto. Mas, além de bom valor nutricional, o alimento também tem que ser saboroso e atender às expectativas do consumidor. Esta aceitação é parte importante no desenvolvimento ou melhoramento do produto (Barbosa et al., 2016). Diante do exposto, o presente estudo teve o intuito de elaborar uma geleia de menta enriquecida com $S$. platensis e de analisá-la quanto aos parâmetros físico-químicos e microbiológicos, a fím de viabilizar a produção de um alimento diferenciado e inovador quanto aos aspectos nutricionais.

\section{Metodologia}

As análises foram realizadas no Laboratório de Técnica Dietética (LTD), no Laboratórios de Bromatologia e Farmacologia (LBF) e no Laboratórios de Microbiologia e Imunologia (LMI) pertencentes à Faculdade de Ciências Médicas de 
Campina Grande, e outras análises foram realizadas no Laboratório de Bromatologia, do Departamento de Nutrição, do Centro de Ciências da Saúde (CCS) da Universidade Federal da Paraíba (UFPB), campus I, João Pessoa/PB.

\subsection{Obtenção da geleia de menta com Spirulina}

Os ingredientes para obtenção da geleia de menta enriquecida com Spirulina foram adquiridos no comercio local da cidade de Campina Grande/PB, e a Spirulina foi doada pela Fazenda Tamanduá, situada no Município de Santa Terezinha, Estado da Paraíba.

Para a produção da geleia extra de menta foram utilizadas maçãs para a extração da pectina, elaborada na proporção 1:1 (fruta: açúcar) acrescido de 40\% de água. Para tanto, as maçãs e as folhas de menta foram lavadas em água corrente e imergidas em solução sanitizante de água clorada por 15 minutos, na proporção de 100 ppm de hipoclorito de sódio, e, posteriormente, sofreram enxágue. As folhas foram secas com papel absorvente e reservadas. Em seguida, a fruta foi picada com casca. Acrescido o açúcar e a água, assim, levando ao fogo brando $\left( \pm 180^{\circ} \mathrm{C}\right)$ até a formação da geleia, ou seja, atingindo 65 ${ }^{\circ}$ Brix, concernente com a legislação específica, que exige no mínimo $62{ }^{\circ}$ Brix (Brasil, 1978).

Chegando ao ponto de geleia, foi adicionado a folha da hortelã com o intuito de extrair a menta presente nela, sendo acrescidos pedaços triturados. Logo em seguida, depois de pronta a geleia, foram acrescidas diferentes formulações da Spirulina. O processo de elaboração da geleia foi descrito no fluxograma apresentado na Figura 1. Após elaboração, a geleia foi acondicionada em embalagens de vidro, codificada e conservada na geladeira.

Figura 1 - Fluxograma de elaboração da geleia de menta.

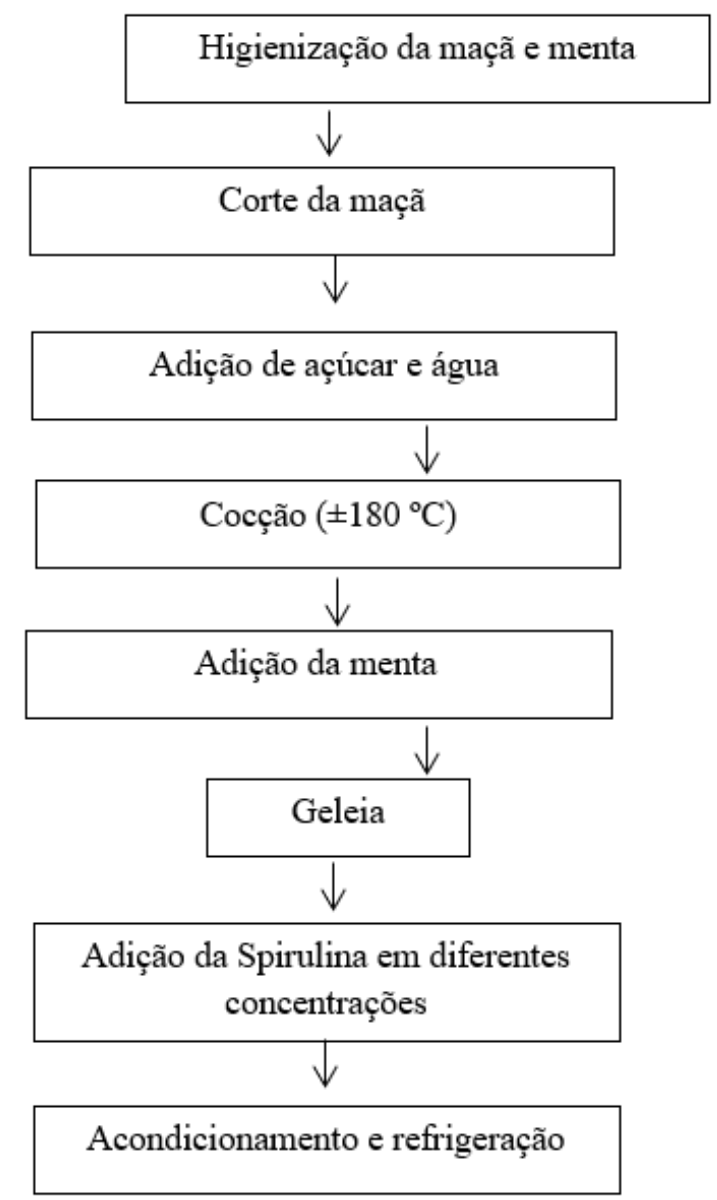

Fonte: Autores (2021). 
A partir deste ponto foram elaboradas quatro formulações de geleia com a adição da Spirulina nas seguintes concentrações: GP amostra padrão (sem Spirulina), GS1 com 1,45\%, GS2 com 2,86\% e GS3 com 4,23\%, de Spirulina. Os ingredientes utilizados para elaboração destas e suas concentrações estão melhor descritas na Tabela 1. Como a geleia é extra a proporção de açúcar usada é a mesma de fruta, como dito anteriormente 1:1.

Tabela 1 - Formulação da geleia GP, GS1, GS2 e GS3.

\begin{tabular}{lcccc}
\hline Ingredientes & GP & GS1 & GS2 & GS3 \\
\hline Açúcar (g) & 60 & 60 & 60 & 60 \\
Menta (g) & 10 & 10 & 10 & 10 \\
Maçã (g) & 60 & 60 & 60 & 60 \\
Spirulina (g) & 0 & 2,5 & 5 & 7,5 \\
Água (ml) & 40 & 40 & 40 & 40 \\
\hline
\end{tabular}

Fonte: Autores (2021).

\subsection{Caracterizações do produto}

As geleias foram caracterizadas quanto aos parâmetros de teor de água (\%), atividade de água (Aa), cinzas (\%), proteína bruta $(\%)$, teor de sólidos solúveis $(\%)$ e acidez titulável (\%), todas determinadas segundo metodologia descrita pelo Instituto Adolfo Lutz - IAL (2008).

O teor de água foi determinado pelo método de secagem das amostras em estufa a $105^{\circ} \mathrm{C}$ até peso constante. A tomada da atividade de água foi determinada através de leitura direta da amostra em temperatura de aproximadamente $25{ }^{\circ} \mathrm{C}$, em higrômetro Aqualab®, modelo 3TE, fabricado pela Decagon. A quantidade de cinzas foi determinada através da incineração da amostra em mufla aquecida a $550{ }^{\circ} \mathrm{C}$, até a obtenção de um resíduo isento de carvão, com coloração branca acinzentada.

O teor de proteína bruta foi quantificado pelo método Micro-Kjeldahl, que consiste na determinação do nitrogênio total. Para converter o resultado em proteína foi utilizado o fator 6,25.

Determinado através de leitura direta da amostra em refratômetro portátil, modelo RTA-100 (escala de 0 a $95{ }^{\circ}$ Brix), colocando uma gota da solução no prisma e fazendo a leitura direta com correção da temperatura feita através de tabela proposta por IAL (2008).

A acidez total foi determinada pelo método titulométrico que se baseia na neutralização dos íons $\mathrm{H}^{+}$com a solução de hidróxido de sódio $(\mathrm{NaOH})$ 0,1 N, padronizada com ácido oxálico, como titulante e fenolftaleína como solução indicadora (Ial, 2008).

\subsection{Análises microbiológicas}

As análises microbiológicas foram realizadas pela Resolução de Diretoria Colegiada (RDC) n. ${ }^{\circ} 12$ de janeiro de 2001, referente ao grupo alimentar: geleia e similares. Os micro-organismos avaliados foram os Coliformes totais e Salmonella sp/25g, todas as análises seguiram as metodologias proposta por Silva et al. (2017).

A confirmação da presença de coliformes totais foi o proposto por Silva et al. (2017) para contagem de coliformes totais/coliformes termotolerantes/E.coli em água e alimentos. Com aplicação da técnica dos tubos múltiplos com preparação de diluições até a $10^{-3}$ em água peptonada. Em seguida, a amostra passou por diluição seriada e inoculação em Caldo Lactosado Verde Brilhante (CLBVB), que permite a fermentação do grupo de coliformes. Após, a inoculação os tubos foram levados para estufa à $35^{\circ} \mathrm{C}\left( \pm 2^{\circ} \mathrm{C}\right)$ por 48 horas. Sendo tomados como positivos os tubos com presença do gás preso no tubo de Durham. 
Um volume de $2,5 \mathrm{~g}$ da amostra foram adicionados $22,5 \mathrm{ml}$ de água peptonada tamponada (1\%) para préenriquecimento, e incubados a $35^{\circ} \mathrm{C}\left( \pm 2^{\circ} \mathrm{C}\right)$ por 18 horas $( \pm 2)$. Após, foi transferido $1 \mathrm{ml}$ para $10 \mathrm{ml}$ de Caldo de Tetrationato (TT) e incubada à $35^{\circ} \mathrm{C}\left( \pm 2^{\circ} \mathrm{C}\right)$ por 24 horas $( \pm 3)$. Depois os tubos foram agitados em vórtex, e estriados em placas contendo Ágar Entérico de Hectoen (HE) e Ágar Bismuto Sulfito (BS) em alíquotas de $0,1 \mathrm{ml}$, as quais foram incubadas a $35^{\circ} \mathrm{C}\left( \pm 2^{\circ} \mathrm{C}\right)$ por 18 horas $( \pm 2)$.

\subsection{Tratamento dos dados estatísticos}

Para a avaliação dos resultados referentes às análises físico-químicas foi aplicada a Análise de Variância (ANOVA) e teste de Tukey, utilizando o nível de significância de 5\%, para comparação das médias. Para o cálculo destes dados, utilizou-se o programa - Statistics Analys Systems, versão 8.12 (Sas, 1999).

\section{Resultados e Discussão}

\subsection{Caracterização físico-química das geleias de menta enriquecidas com Spirulina}

Na Tabela 2 estão apresentados os valores médios da caracterização físico-química das geleias de menta enriquecidas com Spirulina.

Tabela 2 - Valores médios das variáveis físico-químicas das geleias testadas.

\begin{tabular}{lcccc}
\hline \multirow{2}{*}{ VARIÁVEIS } & \multicolumn{3}{c}{ GELÉIAS } \\
\cline { 2 - 5 } & GP & GS1 & GS2 & GS3 \\
\hline Teor de água $(\mathrm{g} / 100 \mathrm{~g})$ & $31,92 \pm 0,43^{\mathrm{b}}$ & $37,32 \pm 0,78^{\mathrm{a}}$ & $33,88 \pm 0,29^{\mathrm{b}}$ & $33,82 \pm 0,53^{\mathrm{b}}$ \\
Cinzas $(\mathrm{g} / 100 \mathrm{~g})$ & $0,33 \pm 0,00^{\mathrm{c}}$ & $0,66 \pm 0,01^{\mathrm{b}}$ & $0,99 \pm 0,01^{\mathrm{a}}$ & $0,99 \pm 0,01^{\mathrm{a}}$ \\
Aa & $0,740 \pm 0,00^{\mathrm{a}}$ & $0,717 \pm 0,00^{\mathrm{d}}$ & $0,731 \pm 0,00^{\mathrm{b}}$ & $0,720 \pm 0,00^{\mathrm{c}}$ \\
Acidez total $(\mathrm{g} / 100 \mathrm{~g})$ & $2,70 \pm 0,27^{\mathrm{d}}$ & $5,56 \pm 0,27^{\mathrm{c}}$ & $10,48 \pm 0,95^{\mathrm{b}}$ & $13,65 \pm 0,27^{\mathrm{a}}$ \\
Sólidos Solúveis $(\%)$ & $65,85 \pm 0,35^{\mathrm{b}}$ & $67,25 \pm 0,21^{\mathrm{a}}$ & $67,00 \pm 0,00^{\mathrm{a}}$ & - \\
Proteína $(\mathrm{g} / 100 \mathrm{~g})$ & $0,73 \pm 0,04^{\mathrm{d}}$ & $3,43 \pm 0,05^{\mathrm{c}}$ & $5,83 \pm 0,13^{\mathrm{b}}$ & $8,35 \pm 0,11^{\mathrm{b}}$ \\
\hline
\end{tabular}

a-d Média \pm desvio-padrão com letras minúsculas diferentes na mesma linha diferiram entre si pelo teste de Tukey $(\mathrm{p}<0,05)$ entre os tratamentos. Fonte: Autores (2021).

A amostra GS1 $(37,32 \% \pm 0,78)$ diferenciou-se significativamente das demais amostras, com teor de água maior, porém ainda permaneceu dentro do valor máximo exigido pela legislação brasileira (Brasil, 1978), que é de 38\%. Os níveis baixos de umidade nos alimentos podem indicar uma maior vida útil por dificultar o crescimento microbiano. Falcão et al. (2020) desenvolveram uma geleia de umbu com adição de Spirulina sp. LEB-18 e encontraram menor teor de água (10,66 - 16,79\%), o que pode ter ocorrido pela utilização de um percentual maior de fruta do que de açúcar em relação ao presente estudo.

Lemos et al. (2019) desenvolveram uma geleia mista de jabuticaba e acerola, cujo teor de água encontrado apresentou valor entre 21,41 e 29,94\%. Em uma das formulações, foi apresentado o percentual de 21,41\% devido à casca ter sido adicionada à polpa de jabuticaba; já nas outras formulações, constatou-se variação entre 26,77\% e 29,94\% devido ao uso da associação das polpas de jabuticaba e acerola.

O valor de umidade das geleias deste estudo ficou próximo ao encontrado por Oliveira et al. (2014) ao estudarem geleia tradicional de umbu-cajá (35,51\%), estando semelhante também aos valores reportados por Santos et al. (2012) em geleia de cagaita (35,21\%), Damiani, Asquieri et al. (2012) em geleia mista de araçá com marolo (35,89\%) e Damiani et al. (2012) em geleia de araçá (34,45\%). Porém, em um outro estudo de Alves et al. (2016) com Geleia da casca do melão e Geleia da casca do 
melão com suco de laranja, foi encontrada uma umidade de 51,45\% e 41,14\%, respectivamente, que justificou o valor com outros trabalhos com valores acima de umidade.

Mota (2006) encontrou em seu estudo com geleia de maçã tipo Gala um teor de cinzas de 0,23\%. Os valores encontrados para as geleias neste estudo variaram de 0,33 a 0,99\%. Isto se deve à utilização da polpa da fruta na elaboração da geleia, evitando a perda de minerais, uma vez que estes podem ser resistentes ao processo de cocção aplicado e a presença da biomassa que elevou este parâmetro.

Os valores de cinzas do estudo de Falcão et al. (2020) também foram maiores nas geleias em que houve a adição da Spirulina, que obtiveram os teores entre $0,51 \%$ e $0,87 \%$, tendo sido considerados maiores do que os teores de cinzas nas geléias comerciais de uva $(0,18 \%)$, damasco $(0,25 \%)$, mirtilo $(0,12 \%)$, morango $(0,23 \%)$ (Naeem et al., 2017), compota de bagaço de tomate com baixas calorias (2,03\%) (Belovié et al., 2017) e geleia de extrato de casca de uva (0,29\%) (Amorim et al., 2019).

A atividade de água (Aa) foi inferior a 0,8 devido à adição de grande quantidade de açúcar, assim, sendo considerados seguros no que diz respeito ao desenvolvimento da maioria das bactérias (Amit et al., 2017). Assis et al. (2007) relataram que durante o processamento e o cozimento, a sacarose, na presença de ácido, sofre uma hidrólise (inversão) na qual açúcares redutores (glicose e frutose) são formados, sendo a taxa de inversão dependente da temperatura, do tempo de aquecimento e do pH da solução (mistura).

Falcão et al. (2020) apresentaram os valores de Aa das geleias de umbu entre 0,58-0,61, o que não permite o crescimento de microrganismos. No entanto, outros estudos relataram Aa variando entre 0,92 e 0,96 em geleias de baixa caloria produzidas a partir de bagaço de tomate (Belovié et al., 2017) e geleia de extrato de casca de uva (Amorim et al., 2019), sendo especialmente alta em produtos nos quais a sacarose é substituída por outros adoçantes.

De acordo com as análises demonstradas na tabela 02 , os resultados obtidos em relação à acidez indicam que quanto maior for a quantidade de Spirulina, mais elevada será a acidez. Segundo Jackix (1998), a acidez total da geleia deve estar entre 0,50 e 0,80, tendo em vista que valores acima de 1,00 levarão à exsudação de liquido, ou seja, sinérese. Já para os valores menores do que 0,50 na geleia, formar-se-á uma fraca rede de gel. Ainda de acordo com o mesmo autor, é a concentração hidrogeniônica $(\mathrm{pH})$ que tem maior importância no processo de geleificação, e não a acidez titulável. E para que a geleificação se torne completa, são as diferentes concentrações de sólidos solúveis que devem formar diferentes valores nas faixas de pH.

De acordo com o estudo de Lemos et al. (2019) o teor de acidez da polpa de jabuticaba (1,32\%) indicou valor superior ao da polpa de acerola que obteve como resultado 1,14\%. Provavelmente, essa diferença ocorreu porque a acerola está no estágio de maturação mais avançado que o da jabuticaba, em que nesse estágio, sabores e odores específicos desenvolvem-se em conjunto com o aumento da doçura, com a redução da acidez e da adstringência (Chitarra, 2005).

A Agência Nacional de Vigilância Sanitária (ANVISA), através da Comissão Nacional de Normas e Padrões para Alimentos estabelece que para geleias o padrão de identidade e qualidade referente ao teor de sólidos solúveis é de 62 a $65 \%$ (Brasil, 1978). A amostra GP está dentro dos valores recomendados. GS1 e GS2 foram de encontro com os valores do estudo de Falcão et al. (2020) nas geleias adicionadas de Spirulina $(64,80-68,10 \%)$. Os autores relatam que a utilização da biomassa elevou os teores de Sólidos Solúveis. Isso pode ser comprovado em GS3, que ficou sem resultado pelo fato de ser a amostra com o maior percentual de Spirulina, apresentando cor mais forte, que dificultou a visualização do conteúdo pelo equipamento, lembrando que não foram realizadas diluições das amostras para leitura.

Valores de sólidos solúveis próximos foram encontrados por Lemos et al. (2019), que elaboraram e caracterizaram geleia prebiótica mista de jabuticaba e acerola, 65 a 68\%. Sendo que as geleias de acerola ficaram com valores mais próximos aos $65 \%$, podendo isso ser justificado pelo uso da casca da jabuticaba nas outras geleias. Oliveira et al. (2014), desenvolveram uma geleia tradicional de umbu-cajá, apresentando 68,47\% de sólidos solúveis, semelhante ao de Chauhan et al. (2013) ao 
estudarem geleias de polpa de coco e Ndabikunze et al. (2011) ao desenvolverem geleias com polpa de Uapaca kirkiana. Os autores citados reportaram valores de sólidos solúveis dentro da faixa de 68,5 a $68,8{ }^{\circ}$ Brix.

No presente estudo, os teores de proteína estiveram entre 0,73 e 8,35, apresentando, assim, um elevado teor proteico da primeira formulação (GP) em comparação as geleias com adição da bioalga. Falcão et al. (2020) encontraram valores para os teores de proteína entre $2,38 \%$ e 3,35\%, tendo sido considerados maiores do que os teores de proteína nas geleias comerciais de uva $(0,27 \%)$, damasco $(0,43 \%)$, mirtilo $(0,31 \%)$, morango $(0,41 \%)$ (Naeem et al., 2017), compota de bagaço de tomate com baixas calorias (1,32\%) (Belović et al., 2017) e geleia de extrato de casca de uva (1,21\%) (Amorim et al., 2019).

\subsection{Análises microbiológicas}

Quanto às características microbiológicas, todas as amostras foram consideradas aptas para o consumo, pois não apresentaram crescimento microbiano, indicando uma adequada qualidade higiênico-sanitária das matérias primas e boas práticas de manipulação na confecção das geleias. Na Tabela 3 podemos observar os resultados encontrados neste estudo.

Tabela 3 - Avaliação da qualidade higiênico sanitária das geleias.

\begin{tabular}{ccc}
\hline \multirow{2}{*}{ AMOSTRAS } & \multicolumn{2}{c}{ ANÁLISES } \\
\cline { 2 - 3 } & Coliformes totais à $35^{\circ} \mathrm{C}(\mathrm{NMP} / \mathrm{g})$ & Salmonella spp./25g \\
\hline GP & $<2 \times 10^{2} / \mathrm{g}$ & Ausente \\
GS1 & $<2 \times 10^{2} / \mathrm{g}$ & Presente \\
GS2 & $<2 \times 10^{2} / \mathrm{g}$ & Presente \\
GS3 & $<2 \times 10^{2} / \mathrm{g}$ & Ausente \\
Legislação* & $<2 \times 10^{2} / \mathrm{g}$ & Ausente \\
\hline
\end{tabular}

Segundo a Resolução Normativa ${ }^{\circ} 15 / 78$ que fixa a identidade e as características mínimas de qualidade a que devem obedecer às GELÉIAS DE FRUTAS. Fonte: Autores (2021).

As análises microbiológicas são de extrema importância para verificar as condições higiênico-sanitárias do alimento, os riscos que os alimentos podem apresentar à saúde e quanto esse produto pode apresentar de vida útil (Santos, 2011).

A presença de coliformes totais e Escherichia coli em alimentos processados segundo Silva et al. (2017), é considerada uma indicação útil de contaminação pós-sanitização ou pós- processo, evidenciando práticas de higiene e santificação aquém dos padrões requeridos para o processamento de alimentos.

A maioria dos coliformes são encontrados no meio ambiente, possuindo limitada relevância higiênica. Devido ao fato de os coliformes serem destruídos com certa facilidade pelo calor, sua contagem pode ser útil em testes de contaminações pósprocessamento (Forsythe, 2002).

De acordo com Silva (2012), as contaminações por Salmonella em alimentos acontecem por meio de agentes químicos, físicos e biológicos. Além do solo e da água, a falta de higienização das mãos também é um fator relacionado à contaminação, juntamente com a refrigeração e o armazenamento incorretos do produto.

Nas infecções ligadas às demais Salmonellas também ocorrem quadros de diarreia e vômito, incluindo, além disso, febre moderada, dor abdominal, mal estar geral, cansaço, perda de apetite e calafrios (Brasil, 2019). A manifestação dos sintomas ocorre de 12 a 36 horas após a ingestão do alimento (Galdino et al., 2013) e de acordo com Shinohara et al. (2008) pode perdurar por até 72 horas. Óbitos são raros quando se trata desse tipo de infecção.

O produto é considerado seguro quando não representa risco ao bem-estar do indivíduo. Dessa forma, o estudo da viabilidade dos alimentos deve ser realizado em todas as etapas de formulação, assim como no produto final para garantia de total segurança do consumidor (Santos, 2011). 


\section{Conclusão}

De acordo com os resultados encontrados pode-se afirmar que o mesmo cumpre as recomendações nutricionais e microbiológicas para geleias, porém são necessários outros experimentos para atestar o valor sensorial, como a aceitação do produto e sua intenção de compra, enaltecendo a viabilidade do produto e utilização comercial. É importante ressaltar que o valor proteico da preparação demonstrou relevante aumento dependendo da concentração utilizada. Também houve aprovação por meio das análises microbiológicas, por detectarem valores dentro dos parâmetros adequados, evidenciando a qualidade das geleias, da matéria-prima e do processo produtivo. A geleia de menta enriquecida com Spirulina apresenta propriedades favoráveis e promissoras, podendo ser comercializada. Além disso, trará benefícios à saúde dos seus potenciais consumidores, visto que apresenta um teor proteico elevado (60\%-70\%).

\section{Referências}

Abdel-daim, M. M., Abuzead, S. M. M. \& Halawa, S. M. (2013). Protective role of Spirulina platensis against acute deltamethrin induced toxicity in rats, PLoS One, 8, e72991.

Abe-matsumoto, L. T., Sampaio, G. R. \& Bastos, D. H. M. (2015). Suplementos vitamínicos e/ou minerais: regulamentação, consumo e implicações à saúde. Cadernos de Saúde Pública, 31 (7), 1371-1380.

Alves, A. A., Sales, J. C. R., Bastos, R. A. \& Oliveira, T. O. (2016). Obtenção e caracterização de geleia a partir das cascas do melão com suco de laranja . Congresso Brasileiro de Ciência e Tecnologia de Alimentos, Gramado/RS.

Amit, S. K.., Uddin, M. M.., Rahman R., Islam, S. M. R. \& Khan, M. S. (2017). A review on mechanisms and commercial aspects of food preservation and processing. Agriculture \& Food Security, 6, 51. https://doi.org/10.1186/s40066-017-0130-8

Amorim, F. L., Silva, M. B. D. C., Cirqueira, M. G, et al. (2019). Grape peel (Syrah var.) jam as a polyphenolenriched functional food ingredient. Food Nutrition Journal, 7: 1584-1594. https://doi.org/10.1002/fsn3.981

Assis, M. M. M., Maia, G. A., Figueiredo, E. A. T., Figueiredo, R. W. e Monteiro, J. C. S. (2007). Processamento e estabilidade de geleia de caju. Revista Ciência Agronômica, 38 (1), 46-51.

Barbosa et al. (2016). Desenvolvimento de Cereal Matinal sem Glúten e com Adição da Microalga Spirulina Platensis. Revista Processos Químicos, 10 (20), p. $151-156$.

Belovié, M., Torbica, A., Paijé-lijakovié, I. \& Mastilovié, J. (2017). Development of low-calorie jams with increased content of natural dietary fibre made from tomato pomace. Food Chemistry. 237: 1226-1233. http://dx.doi.org/10.1016/j.foodchem.06.045

Bertoldi, F. C., Santánna, E. \& Barcelos, O. J. L. (2008). Revisão: Biotecnologia. Boletim do Centro de Pesquisa de Processamento de Alimentos, 26 (1).

Brasil (1978). Resolução CNNPA n. 12, de 24 de setembro de 1978. Fixa os padrões de identidade e qualidade para os alimentos (e bebidas). Brasília: Ministério da Saúde. Agência Nacional de Vigilância Sanitária.

Brasil (2001). Resolução RDC no 12, de 02 de setembro de 2001: Aprova o "Regulamento Técnico Sobre Padrões Microbiológicos Para Alimentos". Brasília: Anvisa - Agência Nacional de Vigilância Sanitária. Anvisa - Agência Nacional de Vigilância Sanitária.

Brasil (2019). Surtos de Doenças Transmitidas por Alimentos no Brasil. Secretaria de Vigilância em Saúde (p. 16) Brasilia: Ministério da Saúde. http://portalarquivos2.saude.gov.br/images/pdf/2019/fevereiro/15/Apresenta-- --o-Surtos-DTA---Fevereiro-2019.pdf.

Chauhan, O. P., Archana, B. S., Singh, A., Raju, P. S. \& Bawa, A. S. (2013). Utilization of tender coconut pulp for jam making and its quality evaluation during storage. Food and Bioprocess Technology, 6 (6), 1.444-1.449.

Chitarra, M. I. \& Chitarra, A. B. (2005). Pós-colheita de frutos e hortaliças: Fisiologia e manuseio (2. ed.). Lavras: UFLA.

Damiani, C., Asquieri, E. R., Lage, M. E., Oliveira, R. A., Silva, F. A., Pereira, D. E. P. \& Vilas Boas, E. V. B. (2012). Study of the shelf-life of a mixed araça (Psidium guineensis Sw.) and marolo (Annona crassi ora Mart.) jam. Ciência e Tecnologia de Alimentos. 32(2), 334-343.

Damiani, C., Silva, F. A., Asquieri, E. R., Lage, M. E. \& Vilas boas, E. V. B. (2012). Antioxidant potential of Psidium guinnensis Sw. jam during storage. Pesquisa Agropecuária Tropical, 42(1), 90-98.

Falcão, J. S., Sobral, T. S.., Cruz, L. F. S., Philadelpho, B. O., Santos, J. E. M., Costa, J. A. V., Druzian, J. I. \& Ferreira, E. S. (2020). Protein-enriched umbu (Spondias tuberosa) jam prepared by supplementation with Spirulina sp. LEB-18. Brazilian Journal of Development, 6 (4), $22714-22729$.

Fang, C., Chen, G \& Kan, J (2020). Comparison on characterization and biological activities of Mentha haplocalyx polysaccharides at different solvent extractions. International Journal of Biological Macromolecules 154, 916-928. 
Forsythe, S. J. (2002). Microbiologia da segurança alimentar. Trad. Maria carolina Minardi Guimarães e Cristina Leonhardt - Porto Alegre: Artmed, p 216, 211.

Galdino, V. M. C. A., Melo, R. T., Oliveira, R. P., Mendonça, E. P., Nalevaiko, P. C. \& Rossi, D. A. (2013). Virulência de Salmonella spp. de origem avícola e resistência a antimicrobianos. Bioscience Journal, 29(4).

Ial. (2008). Normas analíticas do IAL: métodos químicos e físicos para análise de alimentos. (5a ed.), Instituto Adolfo Lutz.

Jackix, M. H. (1998). Geleias e doces em massa. Doces, geleias e frutas em calda (teórico e prático). UNICAMP. 85-99.

Krolow, A. C. R (2013). Preparo artesanal de geleias e geleiadas. Pelotas: Embrapa clima temperado, RS. 9 p. (Embrapa clima temperado. Documento, 138).

Lemos, D. M., Rocha, A. P. T., Gouveia, J. P. G., Oliveira, E. N. A., Sousa, E. P \& Silva, S. F. (2019). Elaboration and characterization of jabuticaba and acerola prebiotic jelly. Brazilian Journal of Food Technology, 22, e2018098. https://doi.org/10.1590/1981-6723.09818

Lisboa, C. R., Pereira, A. M. \& Costa, J. A. V. (2016). Biopeptides with antioxidant activity extracted from the biomass of Spirulina sp. LEB 18. African Journal of Microbiology Research, 10 (3), 79-86. http:// dx.doi.org/10.5897/AJMR2015.7760

Mota, R.V. (2006). Caracterização química e física de geleia de amora-preta. Ciências e Tecnologia de Alimentos, 26(3), 539-543.

Naeem, M. N. M., Fairulnizal, M. N. M., Norhayati, M. K et al. (2017). The nutritional composition of fruit jams in the Malaysian Market. Journal of the Saudi Society of Agricultural Sciences. 16, 89-96. https://doi.org/10.1016/j.jssas.2015.03.002

Ndabikunze, B. K., Masambu, B. N., Tiisekwa, B. P. M. \& Issa-Zacharia, A. (2011). The production of jam from indigenous fruits using baobab (Adansonia digitata L.) powder as a substitute for commercial pectin. African Journal of Food Science. 5(3), 168-175.

Oliveira, C. A., Campos, A. A. O., Ribeiro, S. M. R., Oliveira, W. C. \& Nascimento, A. G. (2013). Potencial nutricional, funcional e terapêutico da cianobactéria spirulina. RASBRAN - Revista da Associação Brasileira de Nutrição. 5 (1), 52-59.

Oliveira, E. N. A., Santos, D. C., Rocha, A. P. T. \& Gomes, J. P. (2014). Desenvolvimento, caracterização e Estabilidade de Geleia Tradicional de Umbu-cajá. Revista Brasileira de Fruticultura, 36(3), 640- 651.

Philippis, R., Sili, A., Paperi, R., Vincenzini, M., et al (2001). Exopolysaccharide-producing eyanobacteria and their possible exploitation: A review. Journal of Applied Phycology 13, 293-299.

Richmond, A. (2004). Handbook of microalgae culture: Biotechnology and applied phycology. Blackwell Science Ltd.

Santos, C.B. (2011). Segurança Alimentar e Rotulagem de Alimento Sob a Perspectiva do Código de Defesa do Consumidor Brasileiro. Percurso Acadêmico, $1(2), 327-346$.

Santos, P. R. G., Cardoso, L. M., Bedetti, S. F., Hamaceck, F. R., Moreira, A. V. B., Martino, H. S. D \& Pinheiro-Sant'ana, H. M (2012). Geleia de cagaita (Eugenia dysenterica DC.): desenvolvimento, caracterização microbiológica, sensorial, química e estudo da estabilidade. Revista do Instituto Adolfo Lutz, 71(2), p. 281-290.

SAS. (1999). SAS/STAT user's guide. Version 8, Vol. 2. Cary, N.C., USA, SAS Publishing, 634 p.

Shinohara, N. K. S., Barros, V. B., Jimenez, S. M. C., Machado, E. C. L., Dutra, R. A. F \& Lima, J. L. F. (2008). Salmonella spp., importante agente patogênico veiculado em alimentos. Ciência \& Saúde Coletiva, 13, 1675-1683.

Silva, A. P. A \& Silva, M. A. M. (2013). Aplicação da Tecnologia dos Alimentos na Preservação do Meio Ambiente. Cadernos UniFOA, 8(1) Esp.

Silva, N., Junqueira, A. C. V., Silveira, A. F. N., Taniwaki, H. M., Gomes, R. A. R. \& Okazaki, M. M. (2017). Manual de métodos de análises microbiológica de alimentos e água. (5a ed.), Blucher.

Silva, R. A. (2012). Ciência do alimento: contaminação, manipulação e conservação dos alimentos. (p. 37). Trabalho de Conclusão de Curso (Especialização) - Universidade Tecnológica Federal do Paraná, Medianeira. 